\title{
Technè
}

La science au service de l'histoire de l'art et de la préservation des biens culturels

$44 \mid 2016$

Archives de l'humanité : les restes humains patrimonialisés

\section{Caractérisation par GC/MS de substances naturelles organiques dans des échantillons prélevés sur un écorché du musée de l'Homme}

Characterization of natural organic substances using GC-MS in samples taken from a flayed man in the Musée de l'Homme, Paris

Jean Bleton, Thomas Bonneau, Juliette Langlois, Noëlle Timbart et Alain Tchapla

\section{(2) OpenEdition} Journals

Édition électronique

URL : http://journals.openedition.org/techne/1044

DOI : 10.4000/techne.1044

ISSN : 2534-5168

Éditeur

C2RMF

Édition imprimée

Date de publication : 1 novembre 2016

Pagination : 60-63

ISBN : 978-2-7118-6339-6

ISSN : $1254-7867$

Référence électronique

Jean Bleton, Thomas Bonneau, Juliette Langlois, Noëlle Timbart et Alain Tchapla, « Caractérisation par GC/MS de substances naturelles organiques dans des échantillons prélevés sur un écorché du musée de l'Homme », Technè [En ligne], 44 | 2016, mis en ligne le 19 décembre 2019, consulté le 27 juillet 2020. URL : http://journals.openedition.org/techne/1044 ; DOI : https://doi.org/10.4000/techne. 1044

La revue Technè. La science au service de l'histoire de l'art et de la préservation des biens culturels est mise à disposition selon les termes de la Licence Creative Commons Attribution - Pas d'Utilisation Commerciale - Pas de Modification 4.0 International. 
Jean Bleton

Thomas Bonneau

Juliette Langlois

Noëlle Timbart

Alain Tchapla

\section{Caractérisation par GC/MS de substances naturelles organiques dans des échantillons prélevés sur un écorché du musée de l'Homme}

Characterization of natural organic substances using GC-MS in samples taken from a flayed man in the Musée de l'Homme, Paris
Résumé. Lors de la restauration d'un écorché conservé au musée de l'Homme, des micro-prélèvements ont été effectués sur des parties endommagées pour tenter d'identifier les substances naturelles organiques utilisées par l'auteur afin de réaliser sa préparation anatomique. Les analyses effectuées par chromatographie en phase gazeuse couplée à la spectrométrie de masse ont permis d'obtenir des informations utiles à la fois pour la restauration de l'objet et pour la connaissance du procédé utilisé par l'anatomiste.

Mots-clés. Préparation anatomique, produits d'injection, peintures, caractérisation par GC/MS.
Abstract. In the course of the restoration of a flayed man now in the Musée de l'Homme, Paris, micro-samples were taken from the damaged parts in an attempt to identify the natural organic substances employed in his anatomical preparation. The analyses carried out using gas chromatography, combined with mass spectrometry, enabled us to obtain information that proved helpful both for the restoration of the object and broadening our knowledge of the process used by the anatomist.

Keywords. Anatomical preparation, injection fluids, paints, characterization by GC-MS.

\section{Introduction}

L'écorché « de race éthiopique ou race blanche ", réalisé par Henri Jacquart en 1853, est conservé dans la collection d'anthropologie du musée de l'Homme, au Muséum national d'histoire naturelle (Paris) ${ }^{1}$. Il a été restauré par Thomas Bonneau, dans le cadre de son diplôme de conservation-restauration, à l'école supérieure des Beaux-Arts de Tours. À cette occasion, des micro-prélèvements ont été effectués, sur des parties endommagées, pour tenter d'identifier les substances naturelles organiques utilisées par l'auteur afin de réaliser sa préparation anatomique. Les échantillons analysés sont principalement les produits d'injection qui permettent aux veines et aux artères de conserver leur volume, et les peintures externes qui permettent de les distinguer. Dans cette étude, la chromatographie en phase gazeuse couplée à la spectrométrie de masse (GC/MS), bien adaptée à l'analyse de mélanges complexes, a été utilisée avec deux protocoles analytiques complémentaires.

Le premier ${ }^{2}$ protocole comprend une extraction au dichlorométhane suivie d'une étape de silylation et une analyse à haute température avec injection «On column ». Il permet d'observer l'échantillon en conservant l'intégrité des molécules présentes. Il est absolument nécessaire pour caractériser correctement les résines terpéniques qui contiennent des marqueurs fragiles. Il permet également d'apprécier l'état de conservation des corps gras : huiles, graisses ou cires. Le second protocole comprend une méthanolyse acide suivie d'une étape de silylation et une analyse dans des conditions « classiques » avec injection «splitless ». Il a été mis au point pour la caractérisation des gommes naturelles et des substances tannantes, mais permet également de bien déterminer la nature des corps gras ${ }^{3}$.

\section{Résultats}

\section{Silylation après extraction au dichlorométhane (fig. 1 à 3)}

Les marqueurs du blanc de baleine ${ }^{4}$ (cires C28 à C38) et ceux de la cire d'abeille (cires C40 à C48 hydroxylées ou non, hydrocarbures H25 à H31, esters E24:0 à E34:0) sont présents en proportions variables dans les trois prélèvements.

Il en va de même pour les diterpènes (R). Parmi ceux-ci, $\mathrm{R} 1, \mathrm{R} 2$ et $\mathrm{R} 4$ sont caractéristiques de la résine de mélèze. R3 et $\mathrm{R} 5$ sont communs à toutes les résines de conifères².

Jean Bleton, ingénieur d'études, Lip (Sys) ${ }^{2}$, LETIAM, Université Paris-Sud (jean.bleton@u-psud.fr). Thomas Bonneau, restaurateur du patrimoine, restes humains (thomas0bonneau@gmail.com). Juliette Langlois, assistant ingénieur, département Recherche, C2RMF (juliette.langlois@culture.gouv.fr). Noëlle Timbart, conservateur du patrimoine chargé des Antiquités égyptiennes et orientales et des restes humains, département Restauration, C2RMF (noelle.timbart@ culture.gouv.fr). Alain Tchapla, professeur émérite, Lip(Sys)², LETIAM, Université Paris-Sud (alain.tchapla@u-psud.fr). 
Fig. 1. Matière à injection noire d'une veine. Chromatogramme obtenu après extraction et silylation.

Fig. 2. Matière à injection rouge d'une artère. Chromatogramme obtenu après extraction et silylation.

Fig. 3. Peinture rouge d'une artère.

Chromatogramme obtenu après extraction et silylation.

Fig. 1 à 3. $\underline{\mathbf{x}}$ : nombre d'atomes de carbone, $\mathbf{n}$ : nombre d'insaturations. Ax : alcool; $\mathbf{C x}$ : cire; $\mathbf{C x}, \mathbf{O H}$ : cire hydroxylée ; $\mathbf{D G x}$ : diglycéride ; Dx : ester triméthylsilylique de diacide ; Ex:n : ester triméthylsilylique de monoacide ; $\mathbf{H x}$ : hydrocarbure linéaire ; R1 : épimanool ; R2 : larixol ; R3 : déhydroabiétate de triméthylsilyle ; R4: acétate de larixyle ; R5 : 7-oxo-

déhydroabiétate de triméthylsilyle ; $\mathbf{R} 6$ : 15-hydroxy, 7-oxo-déhydroabiétate de triméthylsilyle ; $\mathbf{S h}$ : marqueur de la gomme laque (shellac); TGx : triglycéride comprenant $\mathrm{x}$ atomes de carbone dans les chaînes acyles ; *: artefact ; ?: non identifié.
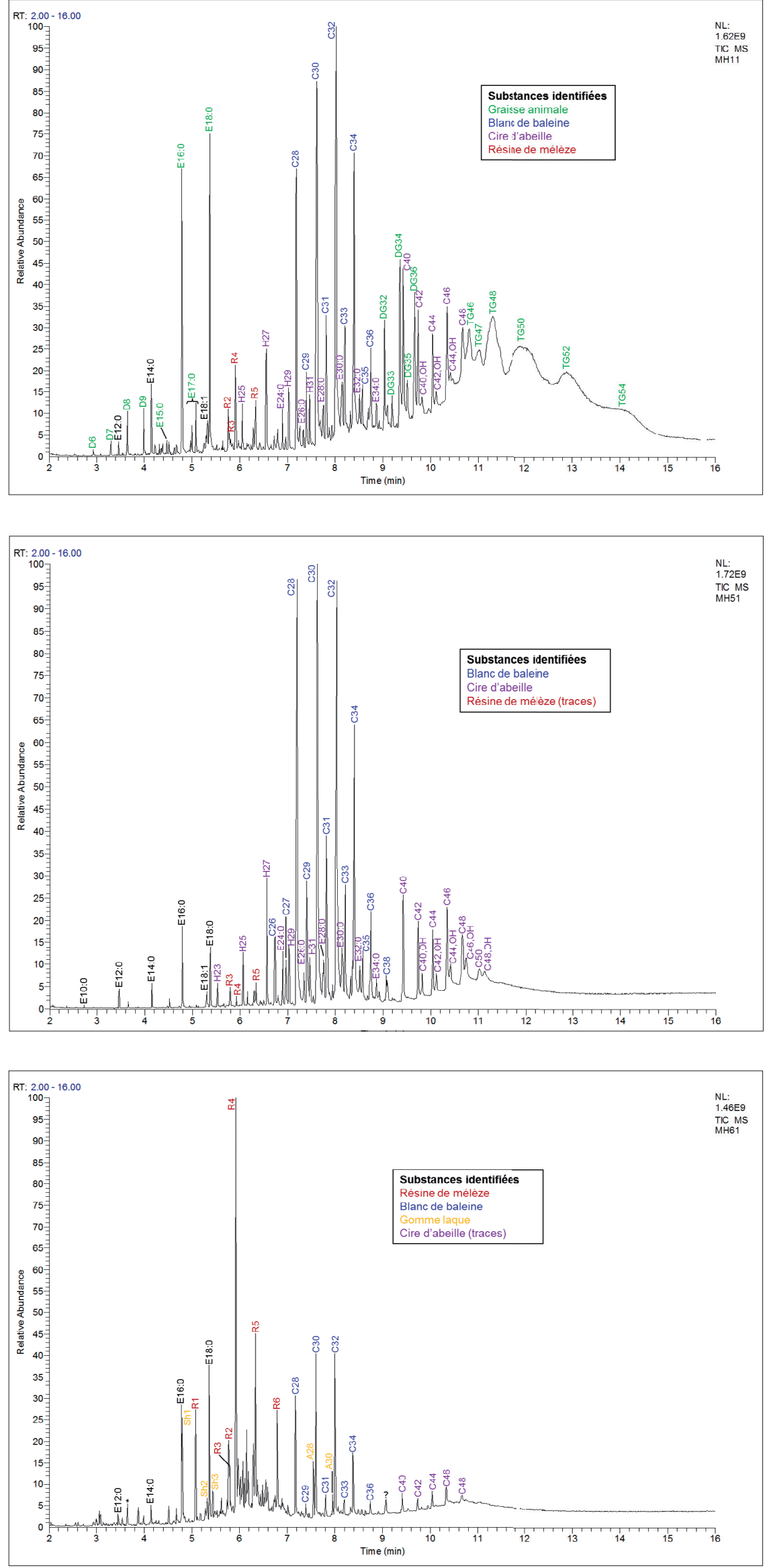

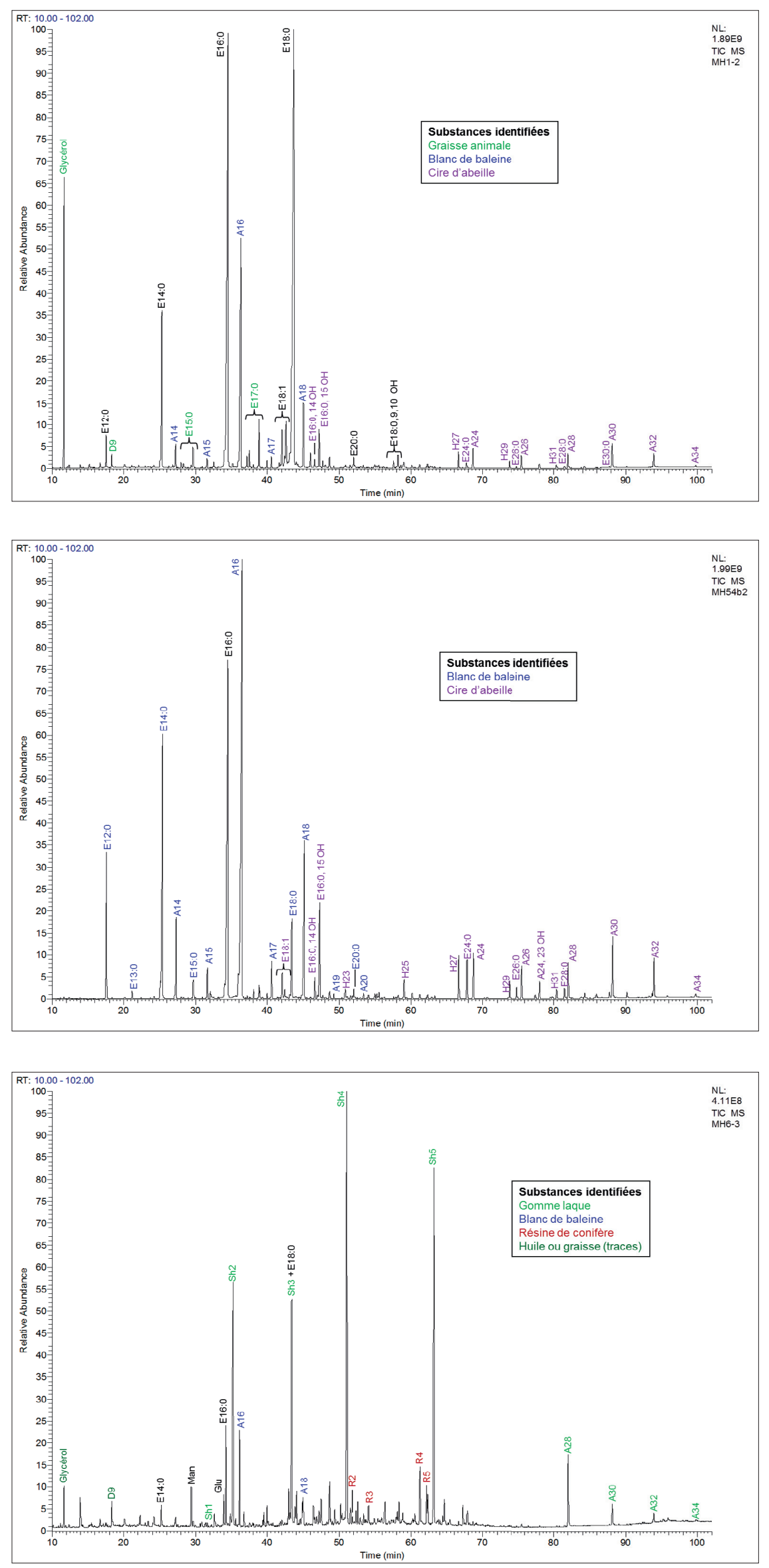

Fig. 4. Matière à injection noire d'une veine. Chromatogramme obtenu après méthanolyse acide et silylation.

Fig. 5. Matière à injection rouge d'une artère. Chromatogramme obtenu après méthanolyse acide et silylation.
Fig. 6. Peinture rouge d'une artère. Chromatogramme obtenu après méthanolyse acide et silylation.
Fig. 4 à $6 . \underline{\mathbf{x}}$ : nombre d'atomes de carbone ; $\underline{\mathbf{n}}$ : nombre d'insaturations. Ax : allcool ; Dx : ester méthylique de diacide ; Ex:n : ester méthylique de monoacide ; E16:0, 140H : ester méthylique hydroxylé sur le carbone 14 ; E16:0, 15OH : ester méthylique hydroxylé sur le carbone $15 ; \mathbf{E 1 8 : 0 ~ 9 , 1 0}$ $\mathbf{O H}$ : ester méthylique hydroxylé en positions $\underline{9}$ et $\underline{10} ; \mathbf{H x}$ : $\underline{\text { hydrocarbure }}$ linéaire ; Glu : glucose ; Man : mannose ; R1 : 7-déhydro-déhydroabiétate de méthyle ; R2 : déhydroabiétate de méthyle ; R3 : déhydroabiétate de triméthylsilyle ; R4 : 7-oxo-déhydroabiétate de méthyle ; R5 : 7-oxo-déhydroabiétate de triméthylsilyle ; Sh : marqueur de la gomme laque (shellac). 
Dans la figure 1, les esters de diacides Dx, les esters de monoacides Ex:n avec $x<22$, les diglycérides DGx et les triglycérides TGx sont les marqueurs d'une huile ou graisse partiellement hydrolysée et peu oxydée. Les esters de monoacides Ex:n avec x impair (15 ou 17) et leurs isomères ramifiés en position iso ou ante-iso permettent d'opter pour une graisse d'origine animale. Enfin, le rapport des quantités relatives de E18:0 (acide stéarique) et E16:0 (acide palmitique) correspond plutôt à une graisse de ruminant.

Dans la figure 3, la présence de gomme laque est révélée par trois marqueurs spécifiques : Sh1 : acide butolique (6-hydroxy-tétradécanoique); Sh2 : acide épilaccilaksholique ; Sh3 : acide épilaccishellolique ${ }^{5}$. Par ailleurs, dans nos conditions opératoires, les alcools gras A28 et A30 ne peuvent provenir que de cette substance.

\section{Méthanolyse acide et silylation (fig. 4 à 6)}

Dans la figure 5, les composés indicés correspondent uniquement au blanc de baleine ${ }^{4}$ et à la cire d'abeille. La quantité relative de E18:0 est faible par rapport à celle de E16:0. En conséquence, dans la figure 4 qui correspond à un mélange contenant également une graisse animale, l'abondance relative de E18:0 par rapport à celle de E16:0 est liée à la présence de la graisse et oriente vers une graisse de ruminant.

Dans la figure 6, les marqueurs de la gomme laque sont majoritaires. Sh1, Sh2 et Sh5 sont respectivement l'acide 6-oxo-tétradécanoique, l'acide 6-hydroxy-tétradécanoique (butolique) et l'acide 9,10,16-trihydroxy-hexadécanoique (aleuritique) ${ }^{5}$. Les composés Sh3 et Sh4 ont été observés dans une gomme laque analysée dans les mêmes conditions, mais leur structure est mal connue. Les alcools A28 à A34 proviennent principalement de cette substance.

De façon générale, les diterpènes Rx sont nettement moins bien observés et les marqueurs spécifiques de la résine de mélèze ont disparu.

\section{Conclusion}

Les deux protocoles se sont avérés complémentaires et nous ont permis d'identifier plusieurs substances naturelles : le blanc de baleine (cire de spermaceti), la cire d'abeille, le suif de mouton, la résine de mélèze (autrefois appelée térébenthine de Venise) et la gomme laque. La résine de mélèze contient des marqueurs spécifiques (larixol et acétate de larixyle), qui ne résistent pas à la méthanolyse acide. Inversement, la gomme laque polymérisée correspond à un polyester naturel de haut poids moléculaire. Le premier protocole permet d'observer des faibles quantités de monomères résiduels, mais la méthanolyse acide permet de transestérifier le polymère et de retrouver des marqueurs spécifiques, comme l'acide aleuritique, en quantités importantes.

Les résultats obtenus nous ont permis d'identifier les substances utilisées par Henri Jacquart pour ses préparations anatomiques et d'orienter différentes étapes de restauration de l'écorché.

La matière à injection des veines contient principalement une graisse de ruminant, probablement du suif de mouton, du blanc de baleine et de la cire d'abeille, en quantités moindres, et un peu de résine de mélèze. La matière à injection des artères ne contient pas de graisse animale. Il s'agit surtout de blanc de baleine additionné de cire d'abeille et d'une très faible quantité de résine de mélèze. La peinture rouge des artères contient principalement de la résine de mélèze et de la gomme laque. Le prélèvement étudié contient certainement également un peu de paroi de l'artère et de matière à injection. Parmi les substances utilisées par Henri Jacquart pour l'injection du système vasculaire, le blanc de baleine est original et n'est pas mentionné dans les traités d'anatomie de l'époque ${ }^{6}$.
Notes

1. Voir l'article de Bonneau et Carminati dans ce numéro.

2. Vo Dhui S., 2007.

3. Tchapla A., 2004

4. Regert M., 2005.

5. Colombini M. P., 2003.

6. Degueurce C., 2008.

\section{Bibliographie}

Colombini M. P., Bonaduce I., Gautier G., 2003, "Molecular Pattern Recognition of Fresh and Aged Shellac", in Chromatographia, 58, p. 357-364.
Degueurce C., Vo Dhui S., Bleton J., Hugon P., Cadot L., Tchapla A., 2008, «Un mystère : la technique de conservation mise en œuvre par Honoré Fragonard pour créer ses fameux écorchés ", Bulletin de la Société Française d'Histoire de la Médecine et des Sciences Vétérinaires 8 , p. 40-57.

Regert M., Langlois J., Colinart S., 2005, "Characterisation of wax works of art by gas chromatographic procedures", Journal of chromatography A, 1091, p. 124136.

Tchapla A., Méjanelle P., Bleton J., Goursaud S., 2004, "Characterisation of embalming materials of a mummy of the Ptolemaic era. Comparison with balms from mummies of different eras", Journal of Separation Sciences, 27, p. 217234.

\author{
Document inédit \\ Vo Dhui S., 2007, « Mise au point \\ de méthodes analytiques pour la \\ caractérisation de la matière organique \\ constituante d'objets du patrimoine \\ culturel », mémoire de thèse \\ de doctorat, Université Paris-Sud.
}

